\title{
Modelo empírico integral de una plantación de Eucalyptus grandis en Concordia, Entre Ríos
}

\author{
Experimental model of a Eucalyptus grandis plantation in Concordia, Entre Ríos
}

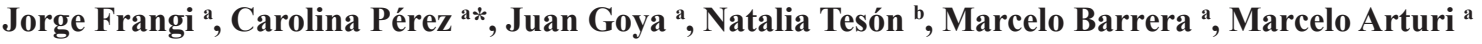 \\ *Autor de correspondencia: ${ }^{\text {a }}$ Universidad Nacional de La Plata, Laboratorio de Investigación de Sistemas Ecológicos \\ y Ambientales (LISEA), Diag. 113 N469, La Plata, Argentina, perezcarolina9017@gmail.com \\ ${ }^{\mathrm{b}}$ Instituto Nacional de Tecnología Agropecuaria (INTA), Estación Experimental Agropecuaria Concordia, \\ Estación Yuquerí, Ruta Provincial 22 y vías del Ferrocarril, Concordia, Entre Ríos.
}

\begin{abstract}
SUMMARY
The Argentinian Mesopotamia is the core of fast-growing tree species plantations of the country. Eucalyptus grandis plantations constitute $90 \%$ of the forested area with Eucalyptus spp. in NE Entre Rios. Based on previous studies on structural and functional features, a comprehensive model is here proposed on emergence of new properties linked to matter and ecosystem processes. Data from different plantations at harvest time were used: biomass, net primary productivity, water flows, nutrient flows, nutrient content in biomass, necromass and soil. Aboveground biomass, productivity and nutrient requirements were higher than belowground ones. Nutrient requirements, except phosphorus, were provided mostly by the absorption rather than by retranslocation whilst the opposite situation was observed in phosphorus. Litter fall and roots mortality were the main ways of nutrient return, except for potassium, which returned mainly by water flows. The high productivity of the plantation was supported by an important water and nutrients use efficiency. The importance of an appropriate harvest residue management is highlighted, since export of total aboveground biomass and its nutrients content involves a high distribution, compared with belowground storages. Compared with nitrogen, high phosphorus retranslocation efficiency would allow plantation to be more independent of mineralization and immobilization edaphic processes.
\end{abstract}

Key words: biomass, necromass, productivity, water flows, nutrient cycles.

\section{RESUMEN}

La Mesopotamia argentina es la zona núcleo de especies forestales de rápido crecimiento del país. Las plantaciones de Eucalyptus grandis constituyen el 90 \% de la superficie forestada con eucaliptos del noreste de Entre Ríos. A partir de estudios previos sobre sus aspectos estructurales y funcionales se propone elaborar un modelo integral del que emerjan nuevas propiedades vinculadas con los almacenajes de la materia y procesos ecosistémicos. Se utilizaron datos de plantaciones al turno, de: biomasa, productividad primaria neta, flujos hídricos, flujos de nutrientes, contenido de nutrientes en la biomasa, necromasa y en el suelo. Se destaca la contribución de la parte aérea de la plantación en la distribución de la biomasa, asignación de la productividad y requerimiento de nutrientes. Los requerimientos de nutrientes, excepto fósforo, fueron provistos mayormente por absorción que por distribución, contrario a lo que ocurrió con el fósforo. La caída al mantillo y mortalidad de raíces fueron las vías principales de retorno de nutrientes, excepto el potasio, el cual retornó principalmente por los flujos hídricos. La elevada productividad de la plantación fue sostenida por una alta eficiencia en el uso del agua y nutrientes. Se destaca la importancia del adecuado manejo de los residuos de la cosecha, ya que la exportación de la biomasa aérea completa y los nutrientes que contiene implican una extracción elevada, comparada con los almacenajes subterráneos. Comparada con el nitrógeno, la elevada eficiencia de redistribución del fósforo permitiría a la plantación mayor independencia de los procesos de mineralización e inmovilización edáficos.

Palabras clave: biomasa, necromasa, productividad, flujos hídricos, ciclo de nutrientes.

\section{INTRODUCCIÓN}

Las plantaciones de Eucalyptus spp. debido a su rápido crecimiento, aptitudes tecnológicas y condiciones de mercado, son las segundas en superficie en la Argentina detrás de las de Pinus spp. La Mesopotamia argentina es su zona núcleo y en ella se destaca el noreste de la provincia de Entre Ríos, donde el 90 \% del área forestada corresponde a
Eucalyptus grandis Hill ex Maiden (MAGyP 2012). Estas plantaciones reemplazan pastizales y bosques abiertos, y su manejo actual provoca cambios en los suelos, la biodiversidad y los ciclos del agua (Carrasco-Letelier et al. 2004, Tesón 2012). Sus impactos negativos podrían reducirse con mejores prácticas.

Los estudios sobre las plantaciones de $E$. grandis en el noreste entrerriano han abordado distintos aspectos: pro- 
ducción volumétrica de madera, biomasa y productividad aérea, biomasa y productividad subterránea, descomposición, balances parciales de nutrientes, balance de agua y nutrientes en los flujos hídricos, suelos, consecuencias del manejo sobre los almacenajes de nutrientes y otros. Algunos de estos estudios destacan la influencia del tipo de suelo sobre la estructura y procesos de las plantaciones de E. grandis. Estas se desarrollan principalmente sobre tres tipos de suelo: arenoso pardo, llamado localmente mestizo, arenoso rojizo profundo y arcilloso. Aquellas sobre suelo mestizo presentan los mayores incrementos medios anuales en términos volumétricos y de biomasa aérea (Goya et al. 1997). Estas plantaciones recuperan nitrógeno y fósforo a través de la redistribución, calculada según Cole y Rapp (1981), pero difieren en cuanto a la eficiencia en el uso de los mismos (Barrera et al. 2005). Las plantaciones sobre suelos arenosos son más eficientes en el uso del fósforo y las que crecen sobre suelos arcillosos lo son en el uso del nitrógeno (Barrera et al. 2005). La duración de los ciclos de cosecha y otros aspectos del manejo, resultan relevantes al evaluar el impacto sobre el almacenaje de nutrientes edáficos. La tala rasa y posterior quema de los residuos de la cosecha como así también el acortamiento de la duración de las rotaciones constituyen los escenarios de mayor impacto negativo sobre los indicadores de sustentabilidad ecológica y económica (Goya et al. 2009). Las tasas de descomposición foliar son bajas para el clima de la zona, lo que puede atribuirse al efecto de la composición bioquímica foliar de los eucaliptos (Goya et al. 2008). También se ha demostrado que la edad de la plantación influye sobre la productividad primaria subterránea neta y en la distribución de la biomasa y el contenido mineral subterráneos (Pérez et al. 2012, 2013). El balance de agua muestra que, de los flujos hídricos aéreos en una plantación al turno, la trascolación constituye la principal fracción de los ingresos de agua al suelo mediante precipitación neta y la transpiración la principal vía de los egresos evapotranspiratorios (Tesón 2012, Tesón et al. 2014). Eucalyptus grandis es capaz de recurrir a la captación foliar de nitrógeno y fósforo de las precipitaciones (ingresos atmosféricos húmedos y secos) (Tesón 2012).

La información producida carece de una interpretación que reúna la totalidad del conocimiento disponible acerca de la estructura y funcionamiento de esas plantaciones. El objeto de este trabajo consiste en elaborar un modelo integral de una plantación de E. grandis al turno, del que emerjan nuevas propiedades o características, vinculadas con los almacenajes de materia seca y los procesos ecosistémicos más relevantes desde el punto de vista de la ecología y manejo del cultivo. Las preguntas que guían dicho objetivo son: ¿Cuál es la distribución de la biomasa, necromasa y sus contenidos minerales en la plantación? ¿Cuál es la asignación aérea y subterránea de fotosintatos y nutrientes? ¿Cuál es la contribución de los subciclos geoquímico, biogeoquímico y bioquímico al ciclo total de cada uno de los nutrientes estudiados? ¿Qué fracción de los requerimientos de nutrientes de la plantación se movilizan por las vías hídricas y cuánto por los procesos ligados a la biomasa? ¿Cuál es la eficiencia de la plantación en el uso de nutrientes y el agua? ¿Cuál es el impacto sobre los suelos del manejo actual?

\section{MÉTODOS}

Este trabajo fue realizado con información provista por diversos estudios ya publicados (cuadro 1). Por lo tanto, toda la información de campo que se indica a continuación es al solo efecto de explicitar en forma sucinta la metodología de los trabajos de referencia.

Área de estudio. Se trabajó con datos de trabajos realizados en plantaciones localizadas en la Estación Experimental Agropecuaria Concordia del Instituto Nacional de Tecnología Agropecuaria (INTA) (31 23' S, 58 02'O), ubicada en Distrito Yuquerí, Departamento Concordia. Los suelos se clasificaron como Fluventic Haplumbrept (Inceptisol), localmente denominado "mestizo" y Oxic Quartzipsamment (Entisol), localmente denominado "arenoso rojizo profundo". Ambos tipos de suelos son de textura arenosa dominante. El clima es templado cálido sin estación seca, con temperatura media anual de $18,7{ }^{\circ} \mathrm{C}$ y precipitación media anual de $1.345,3 \pm 287,7 \mathrm{~mm}$.

Metodología. Los métodos son los que se indican en el cuadro 1. Los datos presentados corresponden a promedios de muestreos realizados en tres parcelas de plantaciones de Eucalyptus grandis de procedencia sudafricana. Todas las plantaciones estudiadas eran de primera rotación que reemplazaron pastizales utilizados como pasturas, recibieron los mismos tratamientos silviculturales y presentaron densidades finales que variaron entre los 870 y 1.111 árboles ha ${ }^{-1}$. El primer valor de densidad corresponde a las parcelas sobre suelo mestizo (Goya et al. 1997) y el segundo a la plantación sobre suelo arenoso (Pérez et al. 2013).

\section{Definición de variables y métodos de campo.}

- Biomasa aérea: en tres parcelas de 0,207 ha, en una plantación de 14 años de edad implantada sobre suelos mestizos, se midieron DAP (diámetro a la altura del pecho) y alturas de todos los individuos. Se apearon 22 individuos distribuidos proporcionalmente a las frecuencias diamétricas del rodal. Para obtener las ecuaciones de peso seco y sus estadísticos se utilizaron técnicas de regresión lineal simple. Se distinguieron los siguientes compartimentos: fuste (diámetro $>5 \mathrm{~cm}$ ), ramas entre 1 y $5 \mathrm{~cm}$, ramas menores de $1 \mathrm{~cm}$, hojas y frutos (Goya et al. 1997)

- Biomasa de raíces: el muestreo se realizó en una plantación de 17 años de edad instalada sobre suelos arenosos rojizos profundos. En cada una de las estaciones del año se extrajeron al azar 15 cilindros de suelo de $6,9 \mathrm{~cm}$ de diámetro y $10 \mathrm{~cm}$ de altura hasta $30 \mathrm{~cm}$ de 
Cuadro 1. Resumen de la fuente de datos empleados en la realización del balance de nutrientes en la plantación modelo de E. grandis de primer ciclo en Concordia, Entre Ríos.

Summary of data source used in carrying out the nutrient balances in model, first cycle E. grandis plantation in Concordia, Entre Ríos.

\begin{tabular}{|c|c|c|c|}
\hline Naturaleza de los datos & Edad (años) & Tipo de datos & Fuente \\
\hline Biomasa aérea & 14 & Análisis dimensional & Goya et al. 1997 \\
\hline Biomasa de raíces & 17 & Muestras de cilindros de suelo & Pérez et al. 2013 \\
\hline Mantillo & 14 & Métodos de cosecha & Goya et al. 1997 \\
\hline $\begin{array}{l}\text { Productividad primaria aérea } \\
\text { neta }\end{array}$ & 14 & Sumatoria del IMA más la caída anual de hojarasca & Goya et al. 1997 \\
\hline $\begin{array}{l}\text { Productividad Primaria } \\
\text { subterránea neta }\end{array}$ & 17 & Diferencias de biomasa & Pérez et al. 2012 \\
\hline $\begin{array}{l}\text { Flujos hídricos y balance } \\
\text { hidrológico }\end{array}$ & 14 & Parcelas hidrológicas & Tesón 2012 \\
\hline $\begin{array}{l}\text { Flujo de nutrientes en la } \\
\text { biomasa aérea }\end{array}$ & 14 & Según Cole y Rapp (1981) & Barrera et al. 2005 \\
\hline $\begin{array}{l}\text { Flujo de nutrientes en la } \\
\text { biomasa subterránea }\end{array}$ & 17 & Según Cole y Rapp (1981) & Pérez et al. 2012 \\
\hline $\begin{array}{l}\text { Contenido de nutrientes en la } \\
\text { biomasa aérea }\end{array}$ & 14 & $\begin{array}{l}\text { Análisis químico por compartimiento y cálculo del } \\
\text { contenido mineral }\end{array}$ & Goya et al. 1997 \\
\hline $\begin{array}{l}\text { Contenido de nutrientes del } \\
\text { mantillo }\end{array}$ & 14 & $\begin{array}{l}\text { Análisis químico por compartimiento y cálculo del } \\
\text { contenido mineral }\end{array}$ & Goya et al. 1997 \\
\hline $\begin{array}{l}\text { Contenido de nutrientes en la } \\
\text { biomasa subterránea }\end{array}$ & 17 & $\begin{array}{l}\text { Análisis químico por compartimiento y cálculo del } \\
\text { contenido mineral }\end{array}$ & Pérez et al. 2013 \\
\hline $\begin{array}{l}\text { Contenido de nutrientes del } \\
\text { suelo }\end{array}$ & 11 & $\begin{array}{l}\text { Análisis químico hasta } 60 \mathrm{~cm} \text { de profundidad y } \\
\text { cálculo del contenido mineral }\end{array}$ & Tesón 2012 \\
\hline $\begin{array}{l}\text { Nutrientes en los flujos de } \\
\text { agua }\end{array}$ & 11 & Análisis químico de los flujos de agua & Tesón 2012, Tesón et al. 2014 \\
\hline
\end{tabular}

profundidad (Pérez et al. 2013). Se distinguieron raíces finas (diámetro $<5 \mathrm{~mm}$ ) y raíces gruesas (diámetro $>$ $5 \mathrm{~mm}$ ). Un muestreo previo realizado hasta $50 \mathrm{~cm} \mathrm{de}$ profundidad mostró una distribución exponencial de las raíces, de tal modo que en los primeros $30 \mathrm{~cm}$ se concentraba el $76 \%$ de la biomasa de raíces finas (Luy et al. 1997).

- Biomasa total: suma de la biomasa aérea más subterránea.

- Mantillo: en cada una de las parcelas en las que se muestreó la biomasa aérea se tomaron al azar 10 muestras de $0,25 \mathrm{~m}^{2}$ (30 muestras en total). Incluyó a la capa superficial (horizonte $\mathrm{O}_{\mathrm{L}}$, conocido también como Oi), compuesta por hojas, ramas, corteza y estructuras reproductivas, y cuando está presente, una delgada capa inferior (horizonte $\mathrm{O}_{\mathrm{F}}$, conocido también como Oe) de dichos tejidos en mayor estado de degradación y de mayor antigüedad (Goya et al. 1997).

- Suelo: se muestrearon tres perfiles dentro de la plantación ubicada sobre suelos mestizos y se tomaron muestras para su análisis químico a las siguientes profundidades: $0 \mathrm{~cm}-5 \mathrm{~cm}, 5 \mathrm{~cm}-20 \mathrm{~cm}, 20 \mathrm{~cm}-35 \mathrm{~cm}, 35 \mathrm{~cm}$ $50 \mathrm{~cm}$ y $50 \mathrm{~cm}-65 \mathrm{~cm}$ (Tesón 2012).
- Contenido de nutrientes en la vegetación y el mantillo: se estimaron como el producto de la masa por unidad de área de cada compartimiento de la vegetación o el mantillo multiplicada por la correspondiente concentración del elemento analizado (Goya et al. 1997). El contenido de nutrientes en el suelo se estimó como el producto de la concentración de cada elemento y la densidad aparente de cada horizonte muestreado hasta los $60 \mathrm{~cm}$ de profundidad (Tesón 2012).

- Productividad aérea (PPN aérea): estimada como la sumatoria del incremento en biomasa (IMA) más la caída anual de hojarasca (Goya et al. 1997).

- Productividad subterránea(PPN subterránea): se estimó mediante diferencias de biomasa entre muestreos consecutivos en cinco momentos a lo largo de un año, teniendo en cuenta el diámetro de las raíces: finas $(<5 \mathrm{~mm})$ y gruesas $(>5 \mathrm{~mm})$, aplicando la fórmula de Frangi et al. (1980) ajustada para su aplicación a sistemas subterráneos (Pérez y Frangi 2000, Pérez et al. 2012).

- Productividad total (PPN total): es la suma de la productividad primaria neta aérea más la subterránea.

- Flujos hídricos: en una plantación al turno sobre suelos mestizos se instalaron tres parcelas de medición de 
flujos de agua. Se midieron durante tres años, el primero de los cuales fue de estabilización y luego se hicieron medidas continuas durante dos años que fueron las empleadas en el trabajo (Tesón 2012). Los detalles del instrumental y técnicas de campo para los flujos de agua ya han sido publicados (Tesón et al. 2014). Se midieron la precipitación bruta, trascolación del dosel, trascolación del mantillo, escurrimiento superficial y transpiración y se obtuvieron mediante cálculo las siguientes variables:

$$
\begin{gathered}
\text { Precipitación neta }= \\
\text { Trascolación dosel + Flujo caulinar } \\
\text { Intercepción del dosel }= \\
\text { Precipitación bruta }- \text { Precipitación neta } \\
\text { Intercepción del mantillo }= \\
\text { Trascolación dosel }- \text { Trascolación del mantillo } \\
\text { Evapotranspiración total }= \\
\text { Intercepción del dosel + Intercepción mantillo }+ \\
\text { Transpiración } \\
\text { Percolación }= \\
\text { Trascolación de mantillo }- \text { Transpiración }
\end{gathered}
$$

- Eficiencia en el uso del agua (WUE): se estimó como el cociente entre la productividad total aérea y la transpiración (Stape et al. 2004). En este caso se emplearon dos cálculos, uno utilizando la productividad total aérea y otro con la productividad total.

- Ciclos de nutrientes: los flujos de nutrientes totales incluyen a aquellos vinculados al ciclo de la materia más los vinculados con los flujos hídricos. Los parámetros de los ciclos de nutrientes fueron calculados de acuerdo con Cole y Rapp (1981). El retorno de nutrientes es la cantidad anual de nutrientes que llega al suelo por la caída de hojarasca y mortalidad de las raíces más los nutrientes en la trascolación y el flujo caulinar. La retención es el contenido de nutrientes en la producción anual de madera y raíces gruesas. Los requerimientos son la suma de la retención más el contenido de nutrientes en la producción de estructuras anuales (hojas, ramas finas, frutos, raíces finas). La absorción es la suma del retorno más la retención más los nutrientes que contenía el agua de lluvia y fueron absorbidos en las copas. Estos últimos se estimaron como la diferencia entre la precipitación bruta menos la precipitación neta. La redistribución es la diferencia entre requerimientos y absorción. La redistribución fue corregida por el lixiviado de nutrientes de la copa, cuando este existió. El lixiviado se calculó como la diferencia entre los nutrientes en la trascolación menos los nutrientes que ingresan a la plantación por depositación atmosférica seca y húmeda (precipitación bruta). La trascolación fue corregida por la evaporación, de acuerdo con las estimaciones de Tesón (2012). Al valor de redistribución obtenido se le restó la cantidad de nutrientes lixiviados. La proporción en que se reduce la redistribución a partir de la corrección por lixiviado se utilizó como factor de corrección de los índices de eficiencia de redistribución.

- Cálculos de eficiencia: se estimó la eficiencia de redistribución de nutrientes móviles como la redistribución dividida por los requerimientos, ambos parámetros obtenidos de acuerdo con Cole y Rapp (1981). La eficiencia en el uso de los nutrientes (EUN) fue estimada (1) para la parte aérea de la plantación, como el cociente entre la biomasa aérea y su contenido mineral y (2) para la plantación en su totalidad (aéreo y subterráneo), de acuerdo a con la ecuación propuesta por Berendse y Aerts (1987):

$$
\mathrm{EUN}=\mathrm{A} * \mathrm{MRT}
$$

Donde, $\mathrm{A}=$ la productividad del nutriente, calculada como el cociente entre la PPN total y la cantidad total del elemento en la biomasa y MRT = tiempo de residencia del nutriente, estimado como la cantidad del elemento en la biomasa dividido por la absorción del nutriente. Por lo tanto,

$$
\mathrm{EUN}=\text { PPN/Absorción }
$$

Donde, $\mathrm{PPN}=$ productividad total de la plantación.

Diagramas de emergía: realizados utilizando los elementos de los modelos de emergía de Odum (1971).

\section{Métodos analíticos.}

- Análisis químicos de la vegetación, el mantillo y el suelo: las concentraciones de fósforo, potasio, calcio y magnesio se realizaron con espectrómetro de emisión de plasma Beckman Spectra-scan. Previamente, alícuotas de muestras de vegetación y mantillo ya secadas fueron molidas y tamizadas con malla 20, luego calcinadas en horno de microondas a $500{ }^{\circ} \mathrm{C}$ y las cenizas fueron disueltas para su posterior análisis químico; en el suelo las muestras fueron secadas al aire y tamizadas con malla de $2 \mathrm{~mm}$. Posteriormente fueron secadas a estufa a $40{ }^{\circ} \mathrm{C}$ y tamizadas con malla 20 . Alícuotas de las muestras se digirieron en medio ácido para la determinación de las concentraciones de elementos totales. El carbono y nitrógeno en el material vegetal y suelo fueron determinados mediante combustión seca en un LECO-CNSanalyzer (Goya et al. 1997, Pérez et al. 2013).

- Nutrientes en los flujos hídricos: se determinaron: carbono orgánico mediante Lachat Instruments Model IL550 TOC-TN Analysis System; nitritos por espectrofotométrica del colorante azoico (Hilton y Rigg 1983); nitratos indirectamente, como nitrito por reducción previa con sulfato de hidracina (Hilton y Rigg 1983); amonio por espectrofotometría del azul de indofenol (Mackereth et al. 1989); fosfato por espectrofotome- 
tría del azul de molibdeno (Murphy y Riley 1962); potasio por fotometría de llama; calcio y magnesio por titulación volumétrica con EDTA y murexida y negro de eriocromo $\mathrm{T}$ como indicadores respectivamente (APHA 1985) (Tesón 2012).

Expresión de los resultados: la información reunida se expresa para una plantación modelo resultante de la integración de los datos disponibles.

\section{RESULTADOS}

Almacenajes de nutrientes. La plantación modelo almacenó en la vegetación más el suelo $265 \mathrm{Mg}$ ha $^{-1}$ de carbono, de los cuales $68 \%$ correspondieron a la biomasa aérea y $32 \%$ al suelo mineral + raíces + mantillo. Los restantes elementos nutritivos analizados $\left(\mathrm{kg} \mathrm{ha}^{-1}\right), 4.915$ de nitrógeno, 777 de fósforo, 4.211 de potasio, 15.306 de calcio y 5.784 de magnesio se almacenaron en las plantas más el suelo, de los cuales 2 a $8 \%$ correspondieron a la biomasa aérea, dependiendo del nutriente, y para porcentajes complementarios al suelo + raíces + mantillo.

La biomasa total de la plantación modelo fue $384 \mathrm{Mg}$ $\mathrm{ha}^{-1}$. Esta se distribuyó en un $98 \%$ asignado a tejidos aéreos y $2 \%$ a tejidos subterráneos hasta los $30 \mathrm{~cm}$ de profundidad. A su vez, el fuste almacenó la mayor parte de los elementos nutritivos (63 a $91 \%$, dependiendo del elemento). Las hojas y la corteza del fuste representaron el $8 \%$ de la biomasa total pero constituyeron el 36 a $66 \%$ de los nutrientes analizados (figura 1).
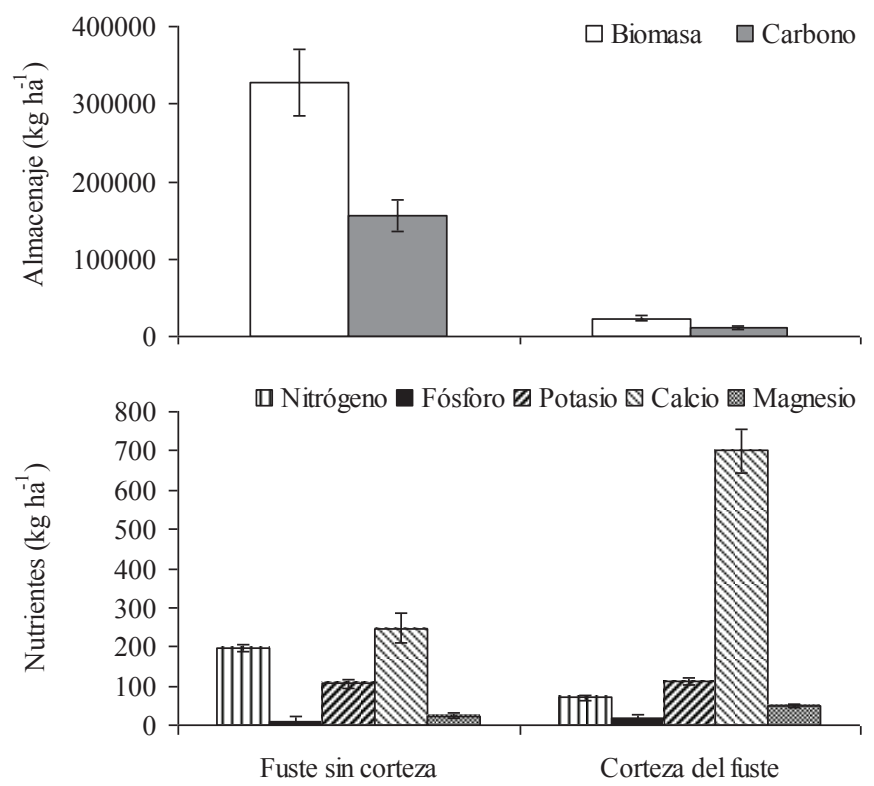

Necromasa y contenido mineral. En las parcelas al turno no existieron ramas muertas en pie, ni ejemplares arbóreos muertos en pie. En consecuencia la mayor parte de la necromasa estuvo constituida por el mantillo y las raíces muertas. El primero representa el $98 \%$ de la necromasa total y 96 a $98 \%$ del contenido mineral de la misma, mientras que las raíces finas muertas aportan los porcentajes complementarios (figura 2). No obstante, al no haber considerado las raíces gruesas muertas se presume que la fracción subterránea podría ser algo mayor.

Productividad primaria neta. La productividad total de la plantación modelo fue $45 \mathrm{Mg} \mathrm{ha}^{-1}$ año ${ }^{-1}$, de los cuales el $96 \%$ correspondió a la productividad aérea y el $4 \%$ a la subterránea (cuadro 2). De la productividad primaria neta total, el mayor porcentaje (60\%) se asignó al crecimiento del fuste, siguiéndole en importancia las hojas (19\%).

Balance hídrico. La precipitación bruta promedio de los períodos de mediciones (2003-2004 y 2004-2005) fue de $1.341 \mathrm{~mm}$. El dosel interceptó $11 \%$ de la precipitación bruta (precipitación neta $89 \%$ ). La mayor contribución a la precipitación neta fue la trascolación (95\% de la precipitación neta), el resto (5\%) descendió externamente como flujo caulinar (cuadro 3). Las salidas gaseosas correspondieron a la suma de las intercepciones de las copas y del mantillo (11 y $14 \%$ de la precipitación bruta respectivamente), y a la transpiración, componente más importante de los egresos gaseosos ( $72 \%$ de la precipitación bruta). La evapotranspiración fue equivalente al 97 \% de la preci-
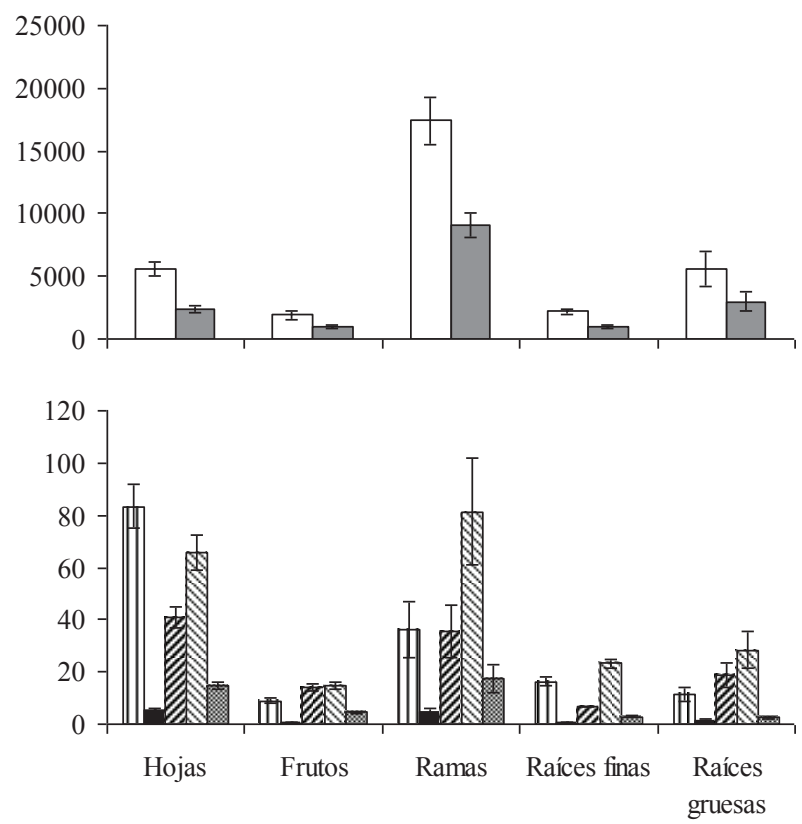

Figura 1. Biomasa y contenido mineral de la biomasa (medias \pm error estándar) de la plantación modelo de E. grandis (14 a 17 años), de primer ciclo en Concordia, Entre Ríos. Gráficos no a escala.

Biomass and biomass mineral content (mean \pm standard error) of model, first cycle E. grandis plantation (14 to 17 years old) in Concordia, Entre Rios. Graphics not to scale. 

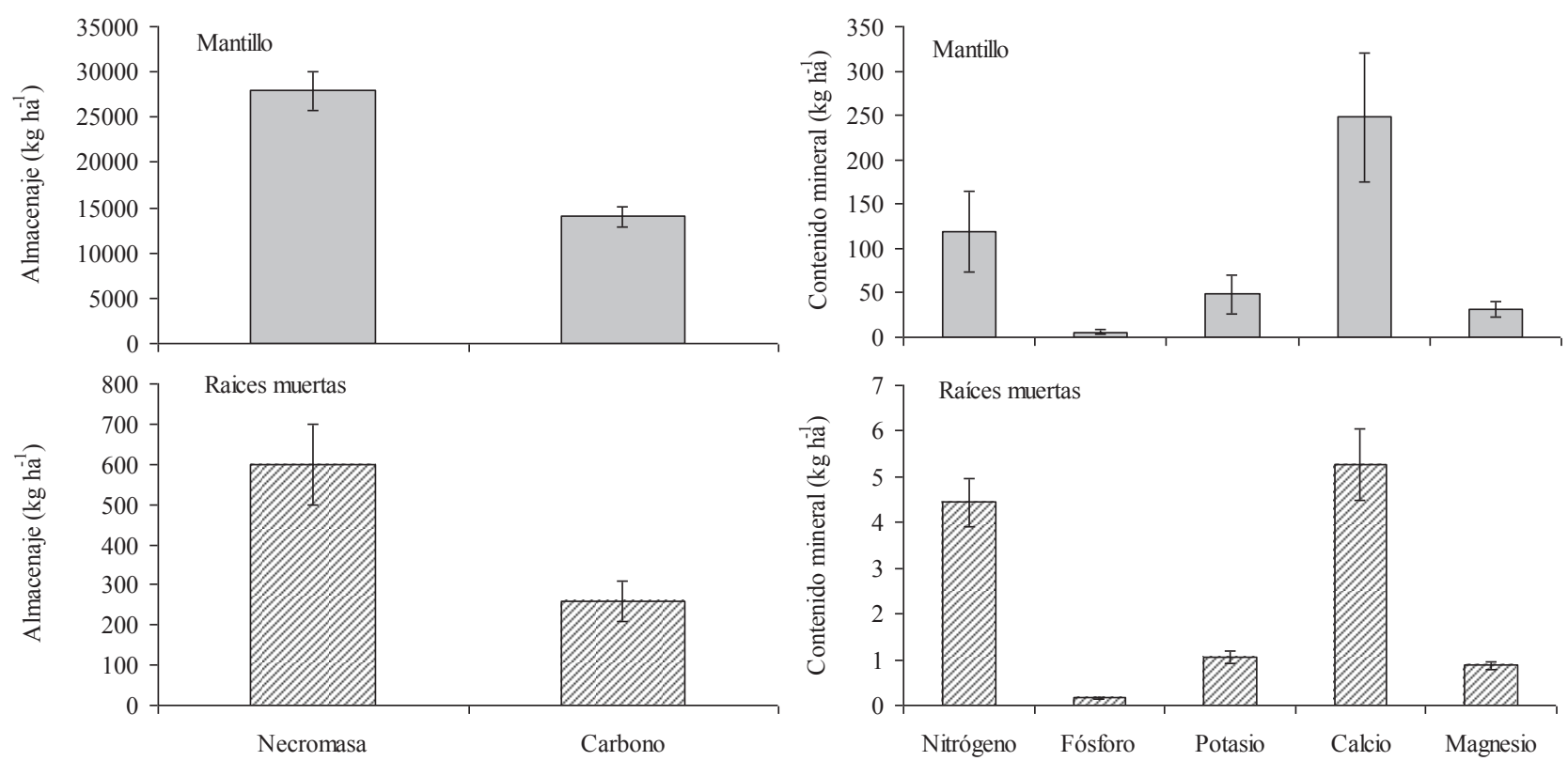

Figura 2. Necromasa y contenido mineral de la necromasa (media \pm error estándar) de la plantación modelo de $E$. grandis (4 años a 17 años), de primer ciclo en Concordia, Entre Ríos. Gráficos no a escala.

Necromass and necromass mineral content (mean \pm standard error) of model, first cycle E. grandis plantation (14 to 17 years old) in Concordia, Entre Rios. Graphics not to scale.

Cuadro 2. Productividad primaria neta aérea, subterránea y total de la plantación modelo de E. grandis (14 a 17 años) de primer ciclo en Concordia, Entre Ríos.

Aboveground, belowground and total (above + belowground) net primary productivity of model, first cycle E. grandis plantation (14 to 17 years old) in Concordia, Entre Rios.

\begin{tabular}{cc}
\hline Compartimentos & $\begin{array}{c}\text { Productividad primaria neta } \\
\left(\mathrm{Mg} \mathrm{ha}^{-1} \mathrm{año}^{-1}\right)\end{array}$ \\
\hline Hojas & $8,42 \pm 0,78$ \\
Fuste & $26,80 \pm 2,40$ \\
Total Aéreo & $43,18 \pm 3,60$ \\
Raíces finas & $0,83 \pm 0,01$ \\
Raíces gruesas & $0,98 \pm 0,01$ \\
Total Subterráneo & $1,81 \pm 0,10$ \\
Total (aéreo + subterráneo) & $44,99 \pm 3,50$ \\
\hline
\end{tabular}

pitación bruta. La eficiencia en el uso del agua fue $4,7 \mathrm{~kg}$ $\mathrm{m}^{-3}$ de agua, considerando la productividad total y $4,5 \mathrm{~kg}$ $\mathrm{m}^{-3}$ de agua considerando sólo la productividad aérea.

Ciclo de nutrientes. Los requerimientos de la plantación se distribuyeron entre 89 y $97 \%$ hacia los tejidos aéreos y entre 3 y $11 \%$ hacia las raíces, dependiendo del nutriente. A su vez, la retención significó aproximadamente $50 \%$ de los requerimientos de carbono, $15 \%$ de los de nitrógeno,
Cuadro 3. Flujos hídricos en la plantación modelo de E. grandis (11 años) de primer ciclo en Concordia, Entre Ríos.

Water flows in model, first cycle E. grandis plantation (11 years old) in Concordia, Entre Rios.

\begin{tabular}{lc}
\hline \multicolumn{1}{c}{ Componente } & $\begin{array}{c}\text { Flujos hídricos * } \\
(\mathrm{mm})\end{array}$ \\
\hline Precipitación bruta & $1.341 \pm 76$ \\
Flujo caulinar & $61 \pm 13$ \\
Trascolación & $1.129 \pm 64$ \\
Precipitación neta & $1.189 \pm 76$ \\
Intercepción copas & $153 \pm 0,5$ \\
Transpiración & $963 \pm 19$ \\
Intercepción mantillo & $192 \pm 40$ \\
Evapotranspiración & $1.307 \pm 58$ \\
Percolación & $34 \pm 18$ \\
\hline
\end{tabular}

* Valores promedio de los períodos 2003-2004 y 2004-2005 (Tesón 2012).

$20 \%$ de los de fósforo, $10 \%$ de los de potasio y magnesio, y $30 \%$ de los de calcio (figuras 3 a 5).

De los requerimientos anuales de carbono, nitrógeno, fósforo, potasio y magnesio de la plantación, la redistribución aportó (\%) 11, 41, 56, 28 y 36, respectivamente, mientras que los porcentajes complementarios fueron obtenidos mediante absorción (figuras 3 a 5). La redistribu- 


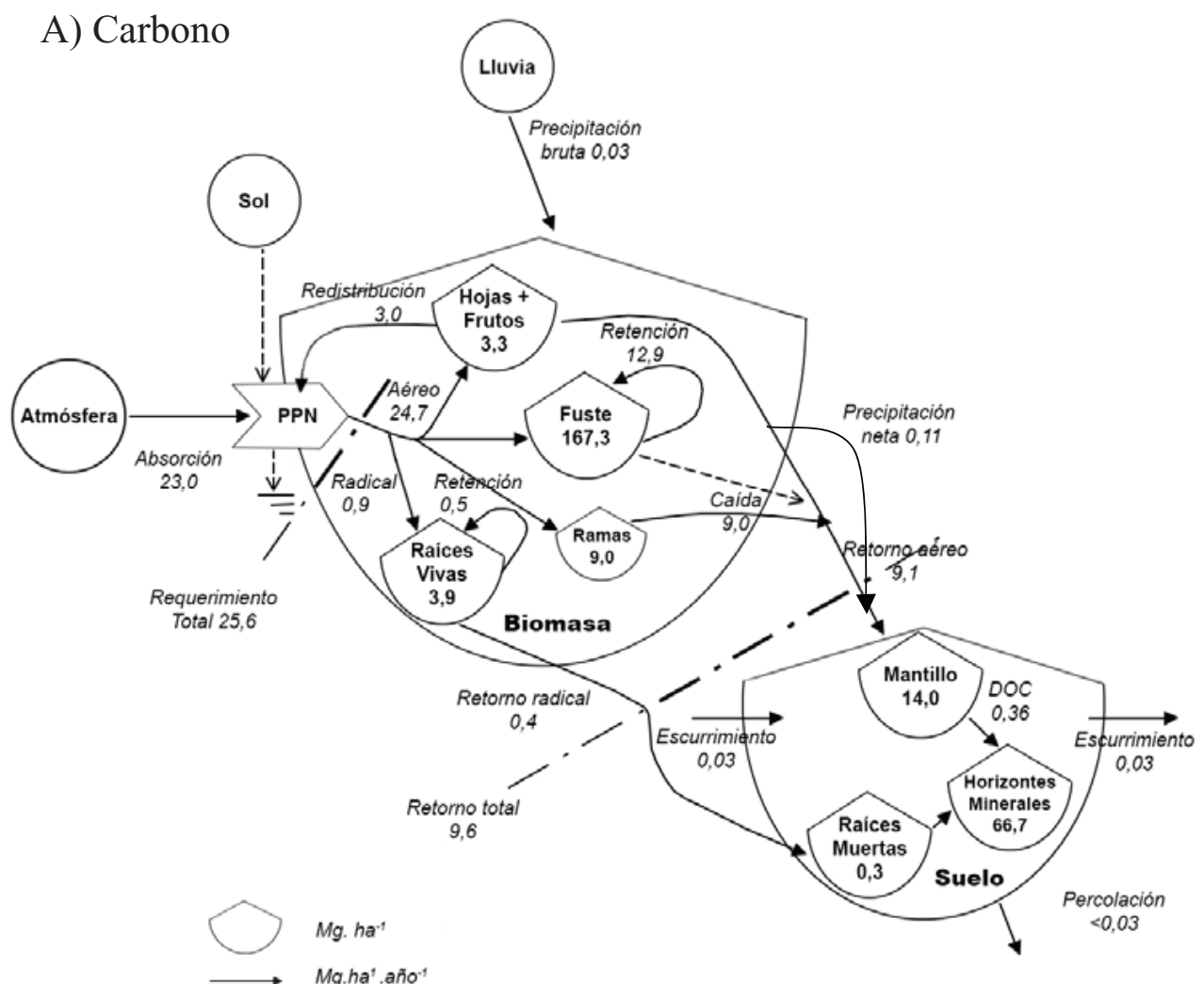

B) Nitrógeno

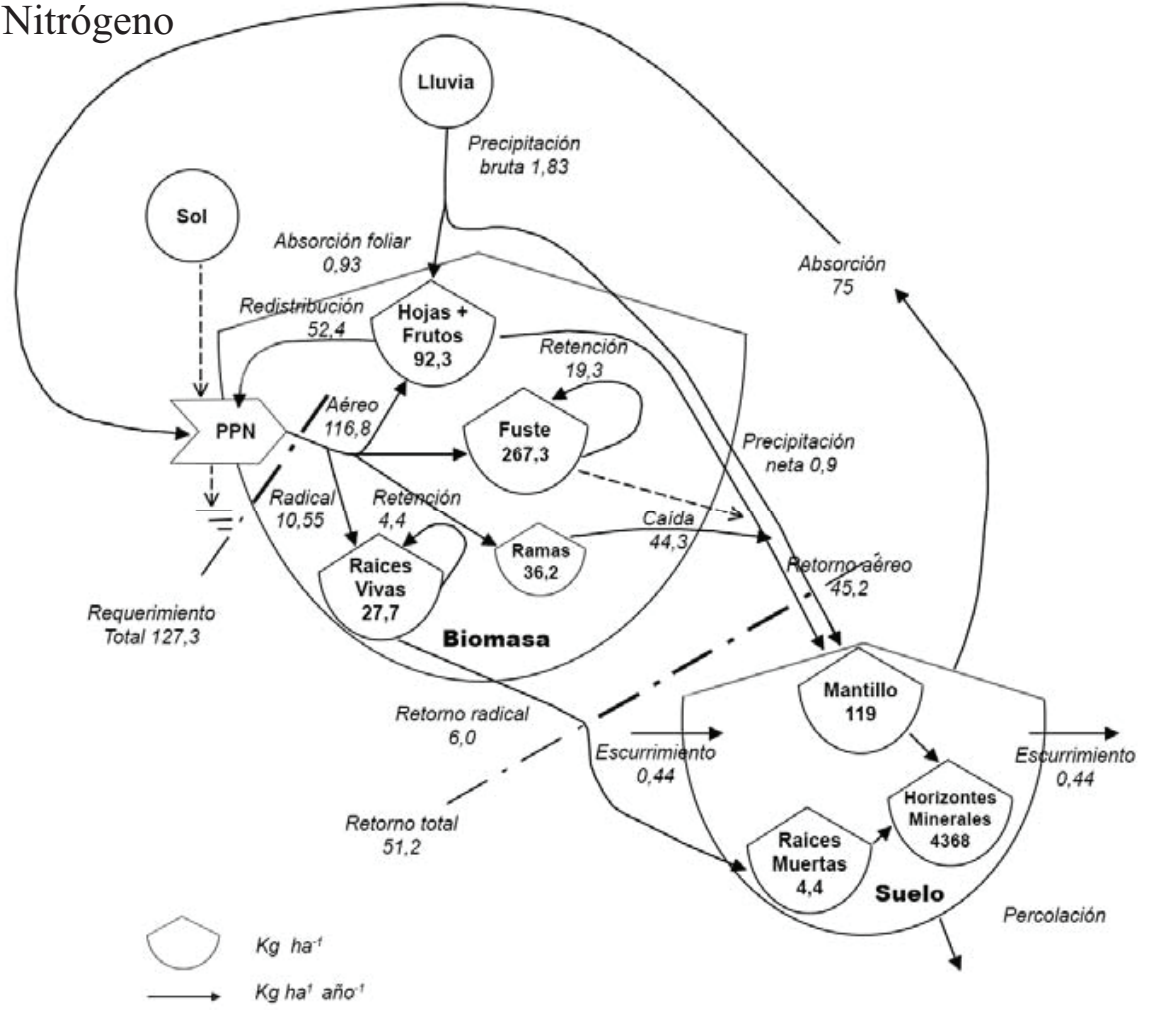

Figura 3. Ciclo del carbono (A) y del nitrógeno (B) en una plantación modelo de E. grandis de primer ciclo, al turno, en Concordia, Entre Ríos. DOC: carbono orgánico disuelto.

Carbon cycle (A) and nitrogen cycle (B) in model, first cycle, at harvest time E. grandis plantation, in Concordia, Entre Rios. DOC: dissolved organic carbon. 


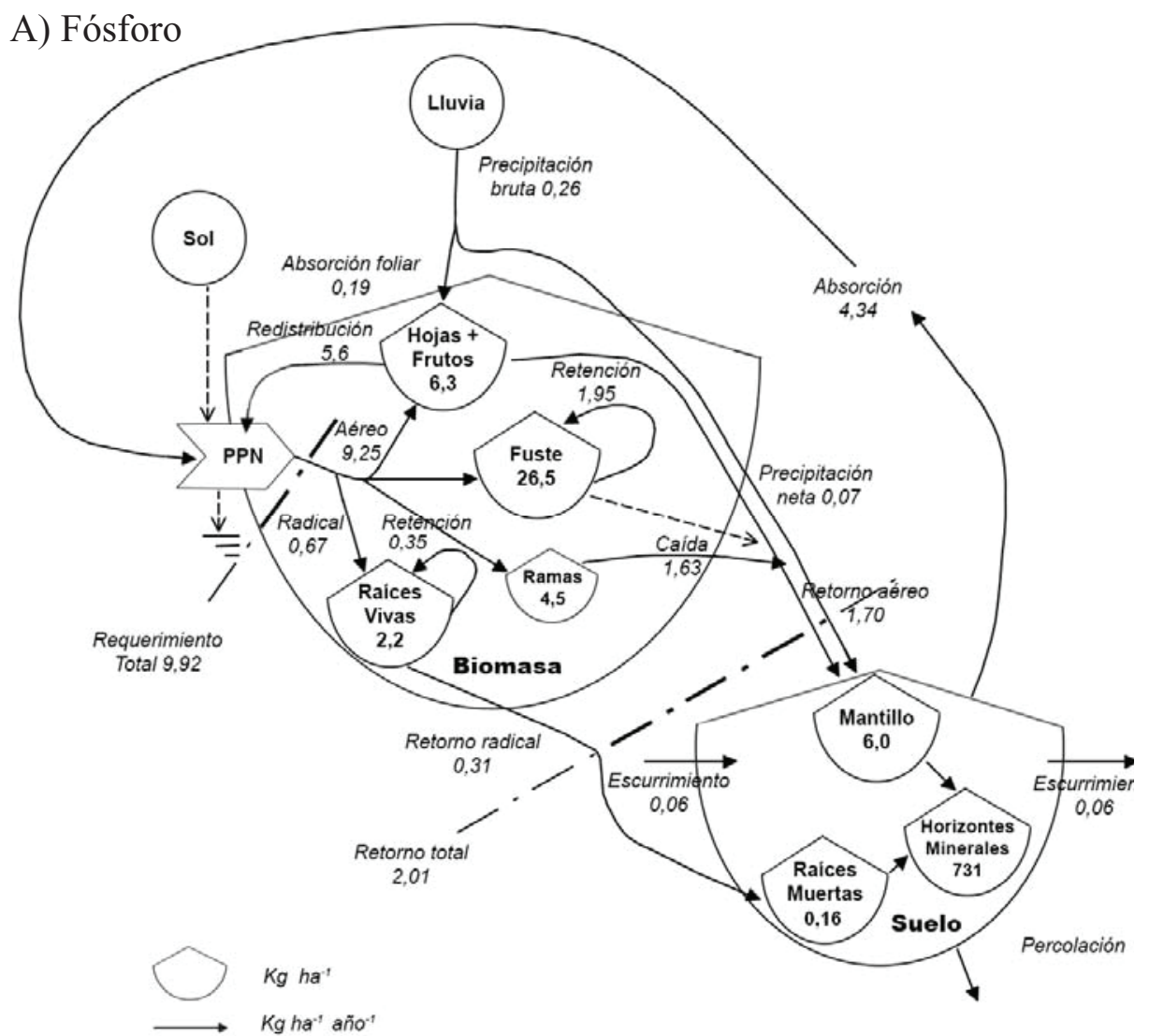

B) Potasio

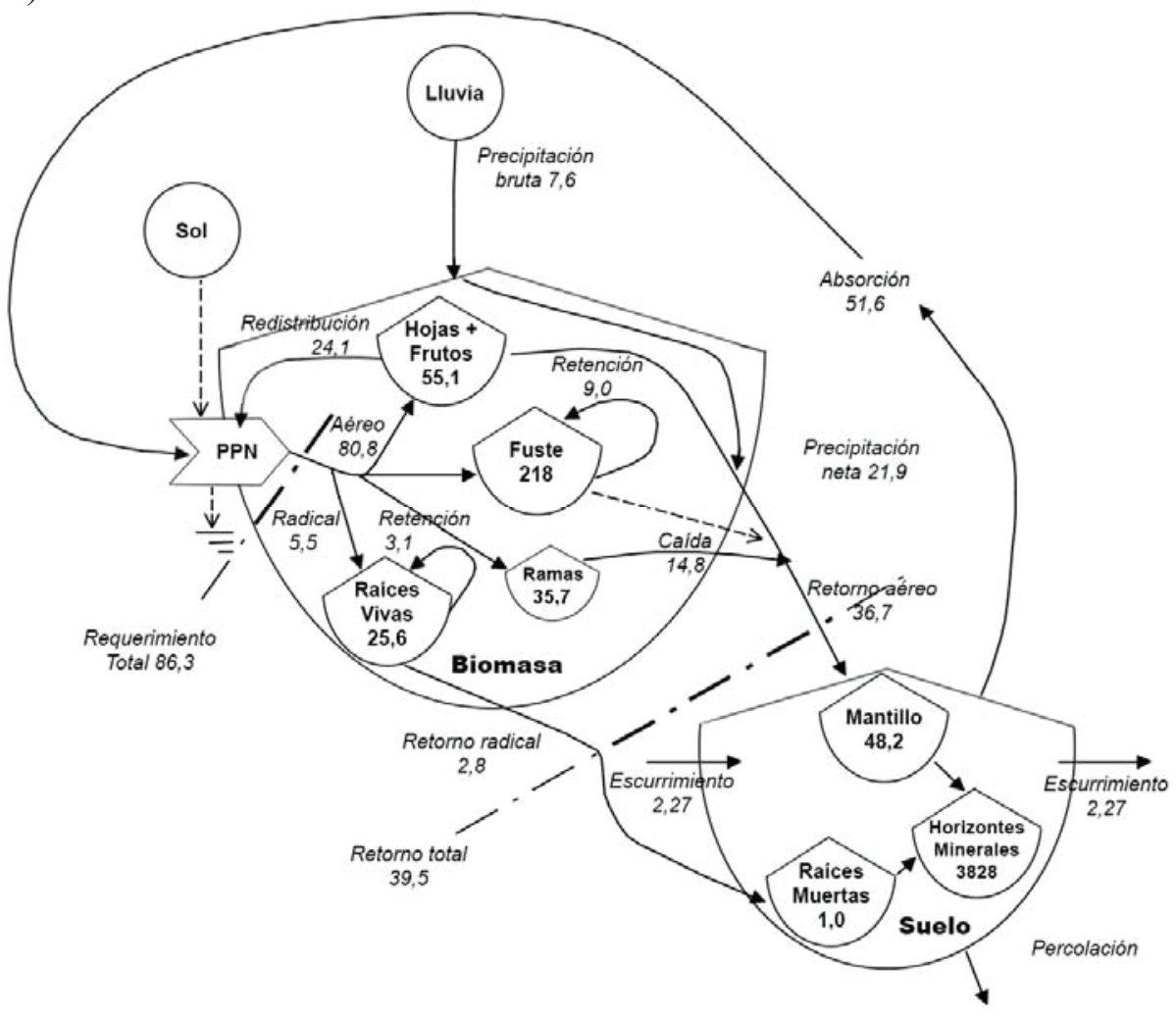

Figura 4. Ciclo del fósforo (A) y del potasio (B) en una plantación modelo de E. grandis de primer ciclo, al turno, en Concordia, Entre Ríos. Phosphorus cycle (A) and potassium cycle (B) in model, first cycle, at harvest time E. grandis plantation, in Concordia, Entre Rios. 


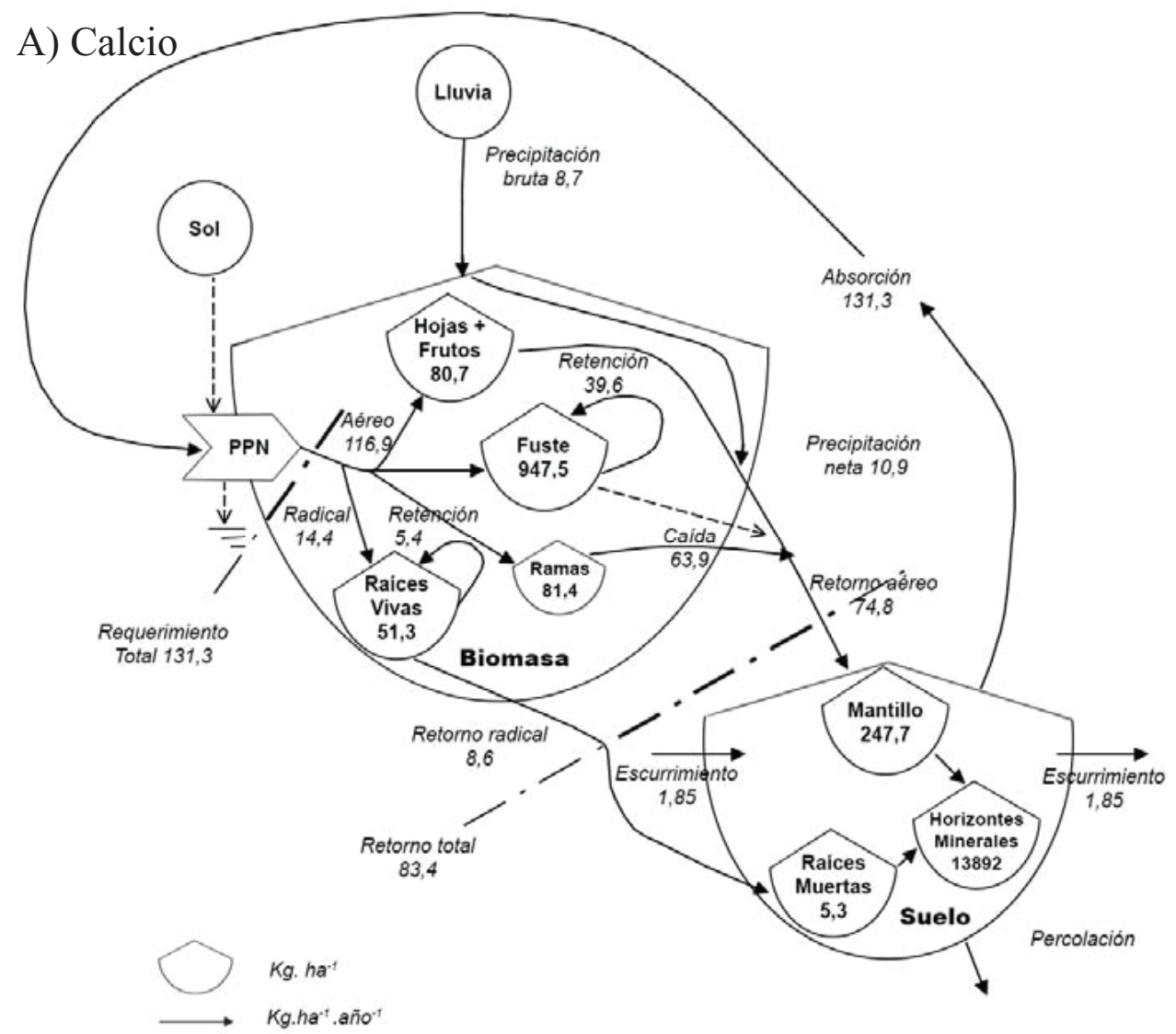

B) Magnesio

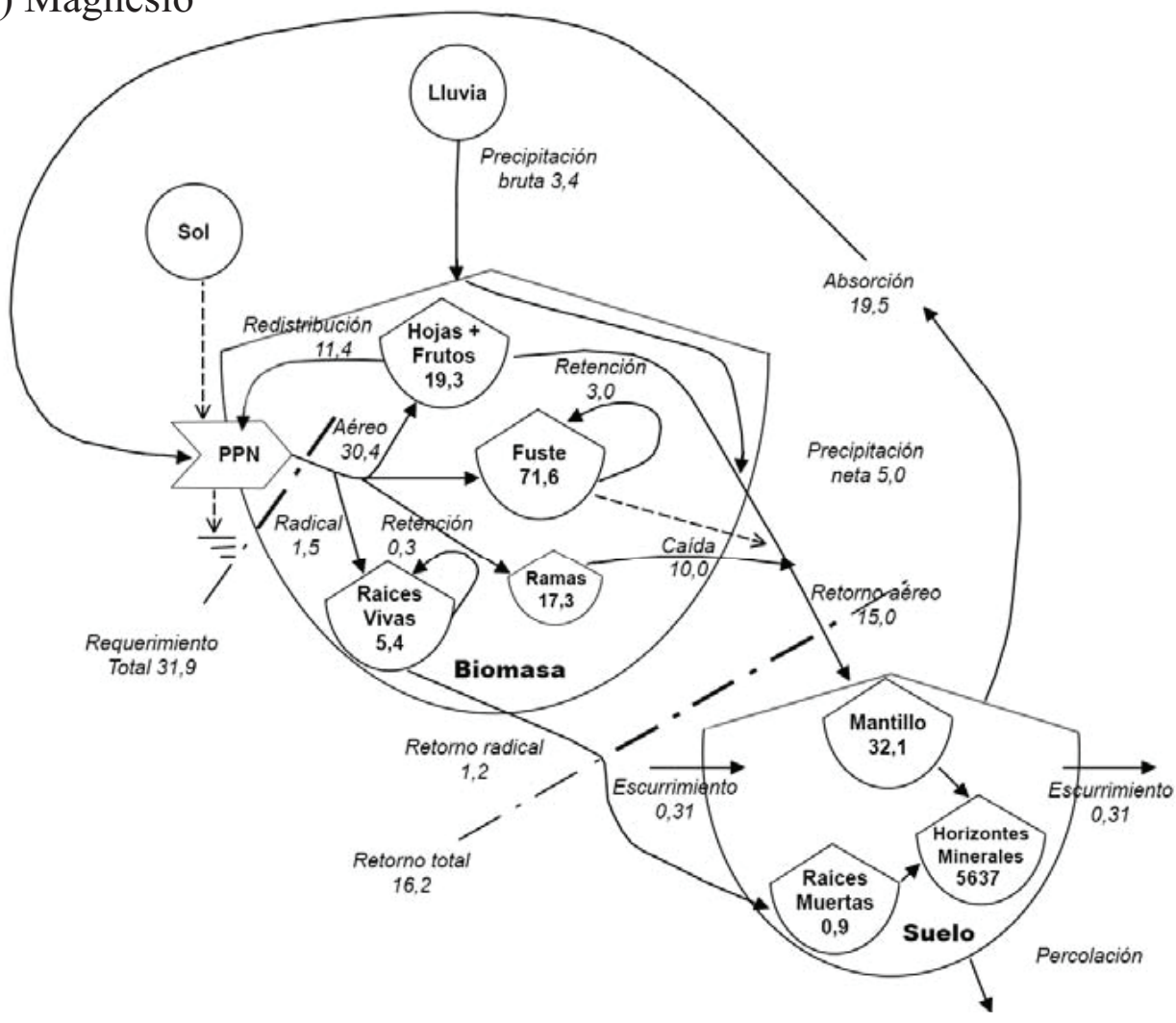

Figura 5. Ciclo del calcio (A) y del magnesio (B) en una plantación modelo de E. grandis de primer ciclo, al turno, en Concordia Entre Ríos. Calcium cycle (A) and magnesium cycle (B) in model, first cycle, at harvest time E. grandis plantation, in Concordia, Entre Rios. 
ción de potasio se redujo en un $30 \%$ a partir de su corrección por lixiviado.

El 1 y el $4 \%$ de la absorción del nitrógeno y fósforo respectivamente, fueron provistos por el agua de lluvia y ocurrió en la copa, atribuyéndose a la absorción foliar. En el caso del calcio la redistribución fue nula y los requerimientos fueron provistos en su totalidad por absorción. La absorción se asignó, según el elemento, entre 85 y 96 \% a los tejidos aéreos y 4 a $15 \%$ a los subterráneos.

El retorno de carbono, fósforo y nitrógeno a través de los restos vegetales representó 97 a $99 \%$ del retorno total y sólo 1 a $3 \%$ se debió al aporte de la precipitación neta. En el caso del potasio, calcio y magnesio los detritos representaron respectivamente el 45,87 y $69 \%$ de dicho retorno y los nutrientes presentes en la precipitación neta aportaron los porcentajes complementarios. La contribución aérea al retorno total, aportada por los restos vegetales más los flujos hídricos varió entre el 85 y $93 \%$, dependiendo del nutriente y los porcentajes complementarios se debieron al aporte de las raíces. El retorno de nutrientes representó 46 a $83 \%$ de la absorción (figuras 3 a 5). Este desbalance entre ingresos y egresos del suelo implican, para esta plantación un déficit $\left(\mathrm{kg} \mathrm{ha}^{-1}\right.$ año $\left.{ }^{-1}\right)$ de 23,7 de nitrógeno, 2,3 de fósforo, 12 de potasio, 48 de calcio y 3,4 de magnesio.

Eficiencia de redistribución y en el uso de los nutrientes. A nivel del ecosistema la eficiencia de redistribución en orden decreciente fue fósforo $>$ nitrógeno $>$ magnesio $>$ potasio. El tiempo de residencia (MRT) en la planta, uno de los componentes de la eficiencia en el uso de los nutrientes (EUN), fue mayor para el fósforo y calcio ( 9 años) comparado con los demás elementos analizados (6 años) (cuadro 4).

\section{DISCUSIÓN}

El suelo, incluyendo las raíces más el mantillo, constituye el principal almacenaje de nutrientes, excepto para el carbono, que se almacena mayoritariamente en la biomasa aérea de la plantación modelada. Comparada con los pastizales que reemplaza, una plantación de $E$. grandis representa un cambio substancial en cuanto a la cantidad y distribución de la biomasa. La biomasa y necromasa de pastizales de Concordia varió entre 6 y $15 \mathrm{Mg}^{2}{ }^{-1}$, de los cuales las raíces representaron 36 a $85 \%{ }^{1}$. Con la implantación de la forestación, la biomasa se incrementa entre 27 y 64 veces, dependiendo del pastizal reemplazado. Además la transformación de ambientes de pastizal natural a plantaciones de $E$. grandis implica un marcado cambio en el cociente raíz:tallo que pasa de 0,3 a 5 , dependiendo del pastizal considerado, a 0,02 en la plantación modelo. El reemplazo de pastizales por plantaciones tiene como efecto disminuciones de carbono y nitrógeno del suelo, atribuidas a una menor calidad de la materia orgánica que ingresa al suelo, una disminución relativa del ingreso de materia orgánica por la vía subterránea, a efectos de la preparación del terreno previo a la instalación de la plantación y a efectos del manejo de los residuos de la cosecha (Goya et al. 2013, Pérez et al. 2013).

La plantación modelada presenta una productividad primaria neta elevada, comparable con los máximos valores reportados para plantaciones de Eucalyptus spp. en Brasil por Stape et al. (2004) y Laclau et al. (2010), que incide en su elevada eficiencia en el uso del agua. El valor de evapotranspiración de esta plantación, $1.307 \mathrm{~mm}$, está dentro del rango observado en plantaciones similares de $E$. grandis en Brasil y en la provincia de Misiones de Argentina, con valores entre 920 y $1.389 \mathrm{~mm}$ (Cristiano et al. 2015). Estos autores determinaron que los valores de evapotranspiración de plantaciones de E. grandis fueron similares a los del bosque nativo del NO de Misiones.

La plantación modelada se caracteriza por una biomasa y productividad aéreas muy superiores a las subterráneas. A su vez, la mayor proporción de la biomasa y de la asignación de la productividad neta están representadas por el fuste. Estas características afectan la distribución de los nutrientes en la vegetación y en consecuencia el efecto de las distintas prácticas de manejo sobre los almacenajes de nutrientes de todo el ecosistema. El impacto de las forestaciones sobre los suelos se ha medido con frecuencia en base al cálculo del contenido mineral aéreo como indicador de la extracción que produce la cosecha del árbol comple-

\footnotetext{
Frangi JL, S Bonaventura, O Bottino, M Ponce. 1978. Estimación de la biomasa de comunidades vegetales del área de la Represa de Salto Grande (Prov. de Entre Ríos). 15 p. Informe inédito.
}

Cuadro 4. Índices de eficiencia de redistribución y de uso de nutrientes (EUN) de la plantación modelo de E. grandis (14 a 17 años) de primer ciclo en Concordia, Entre Ríos. A: productividad primaria neta total/contenido de nutrientes en la biomasa; MRT: contenido de nutrientes en la biomasa/absorción.

Nutrient use and redistribution efficiency indices of model, first cycle E. grandis plantation (14 to 17 years old) in Concordia, Entre Rios. A: total net primary productivity/nutrient content in biomass; MRT: nutrient content of biomass/absorption.

\begin{tabular}{lcrrrr}
\hline Índice & Nitrógeno & Fósforo & Potasio & Calcio & Magnesio \\
\hline Eficiencia de redistribución (\%) & 41 & 56 & 28 & 36 & 3.474 \\
EUN aérea = biomasa aérea/contenido mineral aéreo & 950 & 10.077 & 1.219 & 339 & 3.307 \\
EUN total = A*MRT & 600 & 10.355 & 872 & 350 & 2.307 \\
\hline
\end{tabular}


to, referido en estos casos a la parte aérea de los mismos. Los estudios muestran que las pérdidas ligadas al manejo de las plantaciones varían ampliamente con la duración de la rotación, las fracciones cosechadas y el manejo de los residuos de cosecha (Laclau et al. 2000, Goya et al. 2009). En el presente estudio, la extracción de árbol completo, refiriéndose sólo a la parte aérea, implica una exportación de nutrientes equivalente al 92 a $98 \%$ del total almacenado en la biomasa de la plantación, y la permanencia in situ de los residuos de la cosecha implica una reducción del 39 al $69 \%$ de la exportación de nutrientes. Se destaca la importancia en el sostenimiento de la fertilidad del suelo de aquellas prácticas que aseguren la incorporación al suelo de los residuos de la cosecha, la cual ha sido señalada en otros estudios (Laclau et al. 2000, Goya et al. 2009).

Turner y Lambert (2008) estudiaron plantaciones de E. grandis en New South Wales (Australia) entre los 2 y 30 años de edad. En estas, los requerimientos aéreos de nutrientes alcanzaron un pico a los 8 años, coincidente con el cerramiento de las copas, y se estabilizaron a los 15 años de edad aproximadamente (edad similar a los 14 años de la plantación modelo). Estos requerimientos fueron $\left(\mathrm{kg} \mathrm{ha}^{-1}\right.$ año ${ }^{-1}$ ): 102 de nitrógeno, 7 de fósforo, 65 de potasio, 75 de calcio y 25 de magnesio. La plantación modelada requiere cantidades algo mayores de nitrógeno, fósforo y magnesio pero notablemente mayores de potasio y calcio que la plantación australiana, posiblemente relacionado con la menor biomasa y productividad aérea alcanzada por esta última (271 $\mathrm{Mg} \mathrm{ha}^{-1}$ y $22,4 \mathrm{Mg}_{\text {ha }}{ }^{-1} \mathrm{ano}^{-1}$, respectivamente), aunque también es posible que la eficiencia de uso de los nutrientes sea diferente sobre distintos suelos.

Safou-Matondo et al. (2005) estimaron la eficiencia en el uso de los nutrientes aérea en plantaciones de $E u$ calyptus spp. de 8 años de edad en Pointe Noire, Congo. La plantación modelo muestra mayores eficiencias en el uso del nitrógeno, fósforo y magnesio que las plantaciones más eficientes del estudio de Safou-Matondo et al. (2005), pero menos eficiente en el uso del potasio y calcio.

La eficiencia en el uso de los nutrientes puede ser analizada mediante sus componentes, la productividad del nutriente (A) y el tiempo de residencia del mismo en la planta (MRT) (Berendse y Aerts 1987). De este modo dos especies pueden alcanzar similares eficiencias, pero diferir en los valores de dichos parámetros, respondiendo a diferentes estrategias en el uso de los nutrientes. Una alta eficiencia en el uso de los nutrientes puede lograrse por una elevada productividad del nutriente o por su largo tiempo de residencia. Existen restricciones fisiológicas y morfológicas que impiden que una planta presente valores elevados de los dos componentes del índice. La productividad del nutriente es una característica plástica, que puede variar con la disponibilidad de nutrientes del suelo; en cambio el tiempo medio de residencia del nutriente es una característica inherente a la especie y se mantiene relativamente constante a pesar de los cambios en la disponibilidad edáfica (Aerts y de Caluwe 1994). La mayor eficiencia de E. grandis en la conservación y uso del fósforo puede asociarse a una menor disponibilidad edáfica del nutriente aunque también a características propias de la especie. Los suelos australianos, de donde es originario E. grandis, son extremadamente pobres en fósforo comparados con muchos suelos agrícolas. Los requerimientos de nutrientes de los bosques australianos son alcanzados mediante un conservativo ciclado de nutrientes, a través de una alta eficiencia en la obtención y el uso de los nutrientes (Grove et al. 1996). Comparado con los otros nutrientes analizados en este estudio, el tiempo de residencia del fósforo en la planta es mayor, lo que aumenta la eficiencia con respecto a este nutriente.

En la plantación modelada, los requerimientos de nutrientes son provistos en mayor medida por absorción, a excepción del fósforo, que lo es en mayor medida por redistribución. Respecto del potasio, los ajustes efectuados para diferenciar la redistribución del lixiviado permitieron reconocer que aproximadamente un tercio del potasio que sale de las hojas se moviliza en solución como parte de la precipitación neta, en tanto aproximadamente dos tercios constituye el $28 \%$ redistribuido. De modo que una estimación adecuada de la redistribución de potasio, como de otros nutrientes de fácil lixiviación, requiere tener conocimiento de su circulación por las vías hídricas. En caso contrario, se sobreestima dicho flujo. La eficiencia de redistribución de nitrógeno y fósforo de la plantación modelo se encuentra dentro de los rangos reportados por Laclau et al. (2010) para plantaciones de Eucalyptus spp. en Brasil y Congo. Los mismos autores estimaron una eficiencia de redistribución de potasio entre el 40 y $75 \%$, mayor que el $28 \%$ obtenido luego de la corrección por lixiviado para la plantación aquí estudiada.

Si se tienen en cuenta las diferencias en las vías principales de provisión de nitrógeno y fósforo en la plantación, en el caso del nitrógeno, la mayor contribución de la absorción respecto de la redistribución podría estar explicando, en parte, el descenso del stock de dicho nutriente en el suelo mineral. En el caso del fósforo, la mayor contribución de su redistribución estaría reflejando adaptaciones de los Eucalyptus spp. a los suelos pobres en fósforo de su área de origen (Grove et al. 1996). Sin embargo, a nivel foliar y para las mismas plantaciones de este estudio, Barrera et al. (2005) estimaron que la proficiencia de redistribución de nitrógeno y fósforo fue completa, lo que sugiere la existencia de mecanismos conservativos de ambos nutrientes.

En la plantación modelo las demandas de nutrientes aéreas son superiores a las subterráneas. Sin embargo, las raíces adquirieren mayor importancia cuando se consideran sus aportes al retorno de nutrientes. La relación entre el aporte aéreo y subterráneo al ingreso de materia orgánica al suelo es 20:1 en la plantación modelo, en cambio, cuando se consideran los nutrientes, la relación entre ambos flujos se reduce a un cociente de 5:1 a 9:1, dependiendo del nutriente considerado.

Con respecto a las contribuciones relativas de los tejidos aéreos, subterráneos y los flujos hídricos al retorno, 
se observa que en el caso del nitrógeno, fósforo, calcio y magnesio el mayor aporte es realizado a través de la caída al mantillo y la mortalidad de raíces. Aunque en la plantación aquí estudiada la redistribución de nitrógeno y fósforo contribuye a los requerimientos en una alta fracción, la captura de nitrógeno y de fósforo por vía foliar en las copas indica la avidez de las plantas por obtener el poco nitrógeno y fósforo que se mueve con el agua de lluvia. La captación foliar de nitrógeno ha sido señalada en otras plantaciones de Eucalyptus spp. (Laclau et al. 2010). Ese aporte en la plantación modelada es también bajo, en relación al requerimiento y retorno de nitrógeno y fósforo. En cambio los flujos hídricos representaron la principal vía de incorporación de potasio al suelo y también cobraron importancia, aunque en menor medida, en el caso del calcio y magnesio. La importancia de los flujos hídricos en el retorno de potasio, calcio y magnesio es debida a su enriquecimiento en dichos nutrientes a medida que atraviesa el dosel (trascolación) y el fuste (flujo caulinar), ya que el aporte por la precipitación bruta es muy bajo. Laclau et al. (2010) encontraron patrones coincidentes en los ciclos de nutrientes de plantaciones del Congo y de Brasil, pese a las grandes diferencias edáficas y climáticas entre esas plantaciones. En dichas plantaciones la trascolación más el flujo caulinar representaron entre el 10 y $25 \%$ del nitrógeno y fósforo que ingresan al suelo a través de la caída al mantillo. En el presente estudio la trascolación más el flujo caulinar representan sólo 2 a $4 \%$ del nitrógeno y fósforo que ingresa al suelo por la caída. Esto hace pensar que la baja concentración de nutrientes en aguas de lluvia no contaminadas, sumada a cierta capacidad de recuperación de elementos químicos del agua por parte de las plantas estaría cooperando con la baja circulación de esos nutrientes por la vía hídrica aérea.

A las diferencias en las magnitudes de ingreso de materia orgánica al suelo proveniente de tejidos aéreos y subterráneos, se suman diferencias en los procesos de descomposición y mineralización de los mismos. Las hojas del mantillo de E. grandis presentan una baja tasa anual de descomposición $(k=0,30)$, que determina la acumulación de mantillo, la incorporación lenta de materia orgánica y la inmovilización y baja tasa de ingreso de nutrientes al suelo mineral (Goya et al. 2008). Aunque no se ha estimado en este estudio la descomposición de las raíces, las tasas de renovación de las raíces finas muertas (mortalidad/ necromasa) y de sus almacenajes de nutrientes (retorno subterráneo/contenido mineral de raíces muertas) mostraron una mayor velocidad de recambio comparadas con las de las hojas del mantillo (caída foliar/necromasa foliar y retorno foliar/contenido mineral del mantillo, respectivamente). En las raíces la tasa de renovación de la necromasa fue 1,5 año $^{-1}$, y las del contenido de nutrientes varió entre 1,3 y 2,7 año ${ }^{-1}$, dependiendo del nutriente. En cambio, esas mismas tasas fueron menores en el mantillo foliar $(0,3$ año1). Las bajas tasas de descomposición de los detritos de las plantaciones de Eucalyptus spp. contribuyen a explicar por qué el reemplazo de los sistemas naturales por estas plantaciones tiene como consecuencia un empobrecimiento en materia orgánica y nutrientes de los horizontes minerales superiores, tal como ha sido reportado en varios estudios (Carrasco-Letelier et al. 2004, Tesón 2012, Goya et al. 2013). El cambio en la calidad química del mantillo con la conversión de pastizal a plantación produce una hojarasca de $E$. grandis de menor tasa de descomposición que lleva a un retraso en el aporte de nutrientes al suelo mineral, por lo que durante la primera rotación probablemente la absorción de nutrientes se realiza a partir de los almacenajes procedentes del pastizal reemplazado. Además, en la plantación modelada, dado que el retorno representa una fracción de la absorción de los nutrientes, resulta evidente que los nutrientes necesarios para sostener las altas tasas de crecimiento son provistos por los horizontes minerales a partir del legado de la vegetación natural preexistente.

La silvicultura de esta especie se plantea como una práctica intensiva o semi intensiva, dependiendo de los objetivos y productos a obtener. Los resultados de este trabajo y las consideraciones que se pueden hacer respecto de los posibles impactos de las prácticas silviculturales se refieren a plantaciones de primera rotación y para aquellos regímenes silviculturales que planteen la reforestación después del primer ciclo. En la silvicultura que se considera el manejo del rebrote, que en algunos casos superan las tres o cuatro rotaciones, los efectos negativos se podrían ver atenuados (Goya et al. 2013). Ensayos de fertilización en sitios replantados luego de tres rotaciones y sin remoción de los residuos de la cosecha, no han manifestado respuestas iniciales a la incorporación de diferentes dosis de nitrógeno y fósforo (Graciano et al. 2008).

\section{CONCLUSIONES}

La elevada productividad primaria neta de la plantación modelo de $E$. grandis de primer ciclo es sostenida en parte por una alta eficiencia en el uso del agua, el fósforo y el nitrógeno.

La escasa biomasa relativa de raíces de $E$. grandis reafirma la importancia de un adecuado manejo de los residuos de cosecha, ya que la exportación de la biomasa aérea y los nutrientes que contiene implican una extracción elevada, comparada con los almacenajes subterráneos. Lo cual en sucesivas rotaciones podría implicar impactos negativos sobre la capacidad productiva del sitio.

La importancia del legado edáfico y de nutrientes en el suelo en el primer ciclo, obliga a un manejo cuidadoso del suelo arenoso en las fases de preparación del terreno para plantar y las de cosecha-postcosecha.

El retorno de nitrógeno, fósforo, calcio y magnesio destaca la necesidad de preservar la necromasa del horizonte orgánico, lo que contribuiría a un menor escurrimiento superficial y mayor infiltración que favorece la retención de iones de potasio en solución y su disponibilidad en el suelo. 
La bajísima concentración de nutrientes en la precipitación bruta indica baja contaminación atmosférica e implica que los retornos de algunos nutrientes en los flujos hídricos son debidos a su enriquecimiento durante la trascolación y flujo caulinar.

La avidez en la captura foliar del escaso nitrógeno y fósforo que fluyen disueltos en agua de lluvia por las copas indica el carácter crítico que tienen estos nutrientes para las plantas.

La redistribución del potasio es sobreestimada si no se considera el lixiviado.

Excepto el fósforo, de mayor circulación bioquímica, los elementos circularon más por la vía biogeoquímica.

El fósforo es el elemento con la mayor eficiencia de redistribución, por lo cual la plantación modelo de E. grandis muestra para este nutriente una mayor independencia de los procesos de mineralización/inmovilización edáfica, que el nitrógeno.

Estas conclusiones sobre plantaciones en suelos arenosos en principio no deberían extenderse a plantaciones sobre suelos arcillosos de la misma zona.

\section{AGRADECIMIENTOS}

Este trabajo se realizó con el apoyo de la EEA INTA Concordia, y en el marco del Convenio de Cooperación de Largo Plazo entre el LISEA-UNLP y el International Institute of Tropical Forestry, IITF-USDA-FS, Rio Piedras, Puerto Rico. Agradecemos profundamente a Mary Jeanne Sánchez y personal a su cargo en el Laboratorio Químico de IITF, al Proyecto Forestal de Desarrollo (MAGyP) por la financiación otorgada, como también a Mariana Dabadie, responsable del Laboratorio Químico del LISEA.

\section{REFERENCIAS}

Aerts R, H de Caluwe. 1994. Nitrogen use efficiency of Carex species in relation to nitrogen supply. Ecology 75(8): 23622372.

APHA (American Public Health Association, US). 1985. Standard Methods for the Examination of Water and Wastewater. Washington DC, USA. American Public Health Association. 1268 p.

Barrera MD, JF Goya, JL Frangi. 2005. Ciclo y eficiencia en el uso de N y P en plantaciones de Eucalyptus grandis (Hill ex Maiden) sobre diferentes tipos de suelos en Entre Ríos, Argentina. In Actas del III Congreso Forestal Argentino y Latinoamericano. Corrientes, Argentina.

Berendse F, R Aerts. 1987. Nitrogen-use-efficiency: a biologically meaningful definition? Functional Ecology 1(3): 293296.

Carrasco-Letelier L, G Eguren, C Castiñeira, O Parra, D Panario. 2004. Preliminary study of prairies forested with Eucalyptus sp. at the northwestern Uruguayan soils. Environmental Pollution 127(1): 49-55.

Cole DW, M Rapp. 1981. Elemental cycling in forest ecosystems. In Reichle CE ed. Dynamic properties of forest ecosystems. Cambridge, UK. Cambridge University Press. p. 341-409.
Cristiano PM, PI Campanello, SJ Bucci, SA Rodriguez, OA Lezcano, FG Scholzc, N Madanes, D Di Francescantonio, LO Carrasco, YJ Zhang, G Goldstein. 2015. Evapotranspiration of subtropical forests and tree plantations: A comparative analysis at different temporal and spatial scales. Agricultural and Forest Meteorology 203: 96-106.

Frangi JL, NE Sánchez, MG Ronco, G Rovetta, R Vicari. 1980. Dinámica de la biomasa y productividad primaria aérea neta de un pastizal de «flechillas» de Sierra de la Ventana (Buenos Aires, Argentina). Boletín de la Sociedad Argentina de Botánica 19: 203-228.

Goya JF, JL Frangi, F Dalla Tea, MA Marcó, F Larocca. 1997. Biomasa, productividad y contenido de nutrientes en plantaciones de Eucalyptus grandis en el NE de la provincia de Entre Ríos. In XII Jornadas Forestales de Entre Ríos. Entre Ríos, Argentina.

Goya JF, JL Frangi, CA Pérez, F Dalla Tea. 2008. Decomposition and nutrient release from leaf litter in Eucalyptus grandis plantations on three different soils in Entre Ríos, Argentina. Bosque 29(3): 217-226.

Goya JF, JL Frangi, G Denegri, F Larocca. 2009. Simulación del impacto de diferentes regímenes de cosecha sobre el capital de nutrientes e indicadores económicos en plantaciones de Eucalyptus grandis del NE de Entre Ríos, Argentina. AUGMDomus 1: 1-17.

Goya JF, M Arturi, DM Sandoval, CA Pérez, JL Frangi. 2013. Efecto de las Plantaciones de Eucalyptus grandis sobre el Contenido de $\mathrm{N}$ del Suelo en el NE de Entre Rios. In IV Congreso Forestal Argentino y Latinoamericano. Misiones, Argentina.

Graciano C, JF Goya, M Arturi, CA Pérez, JL Frangi. 2008. Fertilization in a fourth rotation Eucalyptus grandis plantation with minimal management. Journal of Sustainable Forestry 26: $155-169$.

Grove S, B Thomson, N Malajczuk. 1996. Nutritional physiology of eucalypts: Uptake, distribution and utilization. In Attiwill P, M Adams eds. Nutrition of Eucalypts. Melbourne, Australia. CSIRO. p 77-108.

Hilton J, E Rigg. 1983. Determination of nitrate in lake water by the adaptation of the hydrazine-copper reduction method for use on a discrete analyser: performance statistics and an instrument-induced difference from segmented flow conditions. Analyst 108(1289): 1026-1028.

Laclau JP, JP Bouillet, J Ranger. 2000. Dynamics of biomass and nutrient accumulation in a clonal plantation of Eucalyptus in Congo. Forest Ecology and Management 128: 181-96.

Laclau JP, J Ranger, JL de Moraes Goncalves, V Maquere, AV Krusche, AT M'Bou, Y Nouvellon, L Saint-Andre', J-P Bouillet, M de Cassia Piccolo, P Deleporte. 2010. Biogeochemical cycles of nutrients in tropical Eucalyptus plantations: main features shown by intensive monitoring in Congo and Brazil. Forest Ecology and Management 259(9): 1771-1785.

Luy A, JF Goya, JL Frangi. 1997. Distribución de la biomasa aérea y subterránea en plantaciones de Eucalyptus grandis de distintas edades en la región de Concordia, Entre Ríos, Argentina. In Actas II Congreso Forestal Argentino y Latinoamericano. Misiones, Argentina.

Mackereth FJH, J Heron, JF Talling. 1989. Water Analysis: some Revised Methods for Limnologists. Cumbria, UK. Fresh Water Biological Association, Scientific Publication $N^{\circ} 36.120$ p. 
MAGyP (Ministerio de Agricultura, Ganadería y Pesca de la Nación, AR). 2012. Elaboración de un mapa de plantaciones forestales de la República Argentina de actualización permanente. Área SIG e Inventario Forestal. Dirección de Producción Forestal. Ministerio de Agricultura Ganadería y Pesca. Presidencia de la Nación. Consultado 12 nov. 2014. Disponible en http://www.minagri.gob.ar/new/0-0/forestacion/inventario/mapa\%20de\%20planta.html

Murphy J, JP Riley.1962. A modified single solution method for the determination of phosphate in natural waters. Analytica Chimica Acta 27: 31-36.

Odum HT. 1971. Environment, Power and Society. New York, USA. Wiley-Interscience. $331 \mathrm{p}$

Pérez CA, JL Frangi. 2000. Grassland biomass dynamics along an altitudinal gradient in the Pampa. Journal of Range Management 53: 518-528.

Pérez CA, JL Frangi, A Luy, JF Goya, MF Arturi. 2012. Requerimiento y retorno de nutrientes de raíces finas en plantaciones de Eucalyptus grandis. In VII Congreso de Medio Ambiente de la AUGM. Buenos Aires, Argentina. 22 p.

Pérez CA, JL Frangi, JF Goya, A Luy, MF Arturi. 2013. Contenido de nutrientes en las raíces finas y el mantillo de rodales de Eucalyptus grandis de diferente edad en la Mesopotamia Argentina. Bosque 34(3): 303-310.

Safou-Matondo R, P Deleporte, JP Laclau, JP Bouillet. 2005. Hybrid and clonal variability of nutrient content and nutrient use efficiency in Eucalyptus stands in Congo. Forest Ecology and Management 210(1): 193-204.

Stape JL, D Binkley, MG Ryan. 2004. Eucalyptus production and the supply, use and efficiency of use of water, light and nitrogen across a geographic gradient in Brazil. Forest Ecology and Management 193(1): 17-31.

Tesón N. 2012. Balance hidrológico y flujo de nutrientes en plantaciones de Eucalyptus grandis, en Concordia, Entre Ríos. Tesis Doctoral en Ciencias Naturales. La Plata, Argentina. Facultad de Ciencias Naturales y Museo, Universidad Nacional de La Plata: $171 \mathrm{p}$.

Tesón N, VH Conzonno, MF Arturi, JL Frangi. 2014. Dissolved organic carbon in water fluxes of Eucalyptus grandis plantations in northeastern Entre Ríos Province, Argentina. Bosque 35(3): 279-288.

Turner J, MJ Lambert. 2008. Nutrient cycling in age sequences of two Eucalyptus plantation species. Forest Ecology and Management 255(5): 1701-1712.

Recibido: 28.09 .15

Aceptado: 04.01.16 